\title{
Mathematical formulation to predict the harmonics of the superconducting Large Hadron Collider magnets: III. Precycle ramp rate effects and magnet characterization
}

\author{
Nicholas Sammut* \\ CERN, The European Organization for Nuclear Research, CH-1211 Geneva 23, Switzerland, \\ and UOM, The University of Malta, Msida MSD 2080, Malta \\ Luca Bottura, Guy Deferne, and Walter Venturini Delsolaro \\ CERN, The European Organization for Nuclear Research, CH-1211 Geneva 23, Switzerland \\ (Received 20 May 2009; published 27 October 2009)
}

\begin{abstract}
The Large Hadron Collider (LHC) at CERN is equipped with a feed-forward control system known as the field description for the LHC (FiDeL) which is designed to predict the magnetic field and its multipoles, hence reducing the burden on beam based feedback. FiDeL consists of a physical and empirical parametric field model based on magnetic measurements at warm and in cryogenic conditions. It is particularly critical during beam injection when the field decays and at the beginning of acceleration when the field snaps back. It is known that the decay amplitude is largely affected by the powering history of the magnet, particularly by the precycle flattop current and duration and the preinjection preparation duration. Recently, we have collected data that quantify the dependence of the decay amplitude on the precycle ramp rate. This paper presents the results of the measurements performed to investigate this effect, and the method included in FiDeL to model the precycle dependence. With this complete picture of dynamic changes, we finally discuss the effect on the data taken at nominally constant field, along the magnet loadline. We show that a correction for dynamic changes is required for adequate magnet characterization.
\end{abstract}

DOI: 10.1103/PhysRevSTAB.12.102401

PACS numbers: 85.70.Ay, 41.85.Lc, 07.55.Db

\section{INTRODUCTION}

The field description for the LHC (FiDeL) provides a feed-forward control to the machine to reduce the burden on feedback control based on beam diagnostics. It is based on the separation of the different physical contributions that add to the magnetic field and its harmonics in the Large Hadron Collider (LHC) superconducting magnets [1]. FiDeL consists of a parametric model whose parameters have been obtained from magnetic measurements performed in warm and cryogenic conditions between 2002 and 2007, and forecasts the field variations during particle injection, acceleration, and collision within a residual error comparable to commissioning beam control requirements. The predicted field and field errors are used to generate reference ramps for the main and corrector circuits, with the aim to reach an accuracy suitable for the beam diagnostics (orbit, tune, chromaticity) to take over and reach the nominal operation control precision required.

The effects that contribute to the field and its variations have been categorized into two classes treated separately in FiDeL: (i) static components [2] which are reproducible and which are solely dependent on excitation current; (ii) dynamic components [3] which, to a certain extent,

*nicholas.sammut@cern.ch are not reproducible and are dependent on excitation current, time, and powering history.

The magnetic measurements performed on the LHC magnets, mainly using the twin rotating coil measurement system [4], were devoted to the characterization of the above effects and were performed on $18 \%$ of the dipole magnets. Studies on LHC superconducting dipole magnets in the static domain are based on loadline measurements (Fig. 1) which consist of taking two measurements on 15 current plateaus ranging from injection $(760 \mathrm{~A})$ to nominal

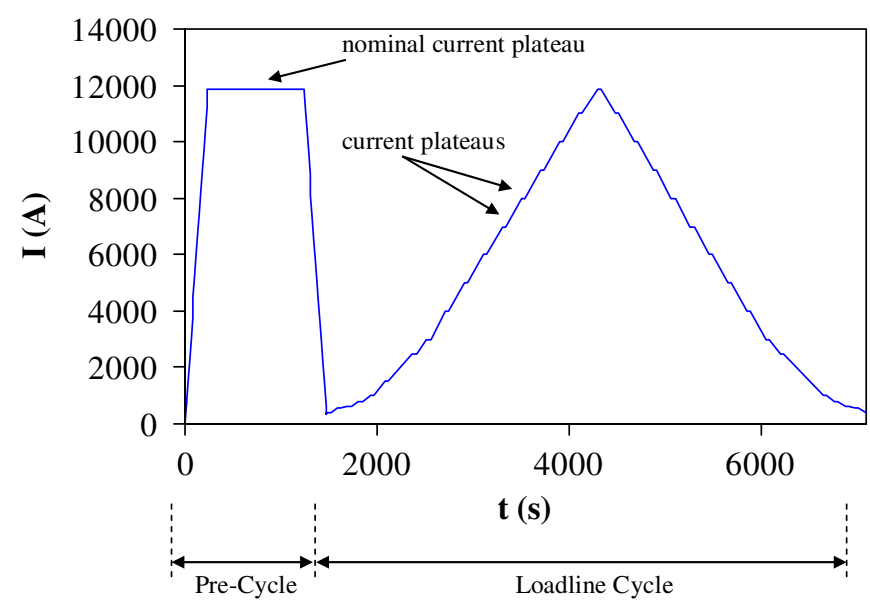

FIG. 1. (Color) The loadline cycle used for series magnetic measurements of the LHC dipoles. 
current (11850 A). The rotating coils [4] perform approximately one measurement every $30 \mathrm{~s}$, i.e., a relatively long time associated with significant field changes during a nominal LHC ramp. The loadline plateaus are intended to avoid having the rotating coils performing a measurement while having a variation of the magnetic field. The data reduction is based on the assumption that the magnetic field and its harmonics have a negligible variation on every loadline current plateau. The measurement results are used to model the magnets in the static domain.

The loadline is preceded by a precycle which is used in tests to reset the persistent currents inside the superconducting filaments and hence put the magnet in a known magnetic state. During machine operation, the precycle is used to achieve a reproducible state. For series magnetic measurements of the LHC dipoles, the precycle ramps up at $50 \mathrm{~A} / \mathrm{s}$ to a nominal current of $11850 \mathrm{~A}$ and then ramps down at the same ramp rate after a waiting time of $1000 \mathrm{~s}$ at this current. Various types of precycle have been experimented for LHC operation, but in all cases the precycle ramps up is at $10 \mathrm{~A} / \mathrm{s}$, limited by the available voltage of the power supply.

The dynamic components (decay and snapback) depend on current and time. They affect the machine mostly at injection and at the beginning of the acceleration. The decay amplitude is known to be strongly dependent on the magnet powering history [3] and especially on the cycle flattop current, the precycle flattop duration, and any waiting time before injection. Decay and snapback were characterized during series tests using measurements performed during simulated LHC cycles [3] which imitate the operating cycle that will be used in machine operation (Fig. 2). For series measurements of dipoles, the magnet is ramped up at $10 \mathrm{~A} / \mathrm{s}$ to the particle injection current $(760 \mathrm{~A})$ with a duration of $1000 \mathrm{~s}$ on the injection plateau. This is followed by the particle acceleration phase in which a standard LHC parabolic exponential linear parabolic (PELP) ramp to the nominal current of $11850 \mathrm{~A}$, which

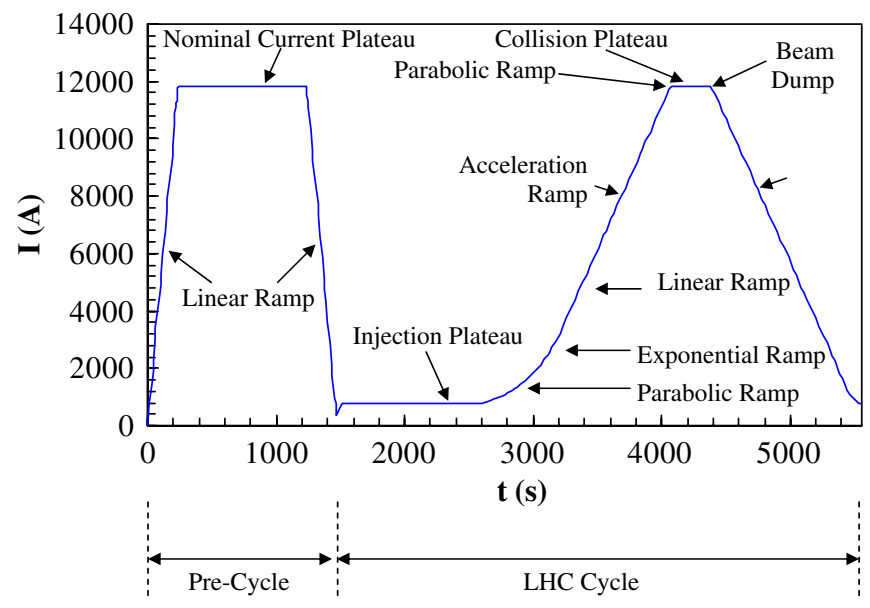

FIG. 2. (Color) The LHC cycle used for series magnetic measurements of the LHC dipoles. corresponds to a bore field of $8.33 \mathrm{~T}$. Being a simulation of the machine operation, the PELP acceleration phase is optimized to: (i) remain within the maximum voltage that the power converters can deliver; (ii) reduce the effects of interstrand coupling currents in the Rutherford cable; (iii) minimize the control bandwidth requirements during particle acceleration; (iv) avoid current overshoots once the magnets reach nominal current.

The magnet is kept on the nominal plateau for $1000 \mathrm{~s}$ after which it is ramped down again to minimum current $(350 \mathrm{~A})$ at $10 \mathrm{~A} / \mathrm{s}$.

The LHC cycle measurements are always preceded by a quench (to erase the memory of previous powering) followed by a precycle with a ramp rate of $50 \mathrm{~A} / \mathrm{s}$ in the case of series magnetic measurements and $10 \mathrm{~A} / \mathrm{s}$ in the case of machine operation.

\section{EFFECT OF PRECYCLE RAMP RATE}

The dependence of decay and snapback on the precycle current, duration, and waiting times before injection has already been presented and discussed elsewhere [3]. We focus here on the influence of the last significant parameter that we identified: the precycle ramp rate. This is especially important because to minimize test time the powering conditions during series magnetic measurements were adjusted, and in some cases they could not be fully representative of operation in the LHC. Specifically, the precycle ramp rate of dipole and quadrupole tests was $50 \mathrm{~A} / \mathrm{s}$ for series tests whereas in the LHC operation, the nominal ramp rate is $10 \mathrm{~A} / \mathrm{s}$, limited by the maximum voltage available over the magnet string of a sector. This results in a variation of decay and snapback as the precycle ramp rate changes the precycle duration. To investigate the magnitude of this effect, we have performed dedicated measurements on four LHC dipole magnets. The measurements were made during standard LHC cycles preceded by precycles of different ramp rates.

Figure 3 shows as an example the decay of the sextupole harmonic $\left(b_{3}\right)$ in dipole MB2598 (aperture 2) for three different precycle ramp rates. From the figure, it can be

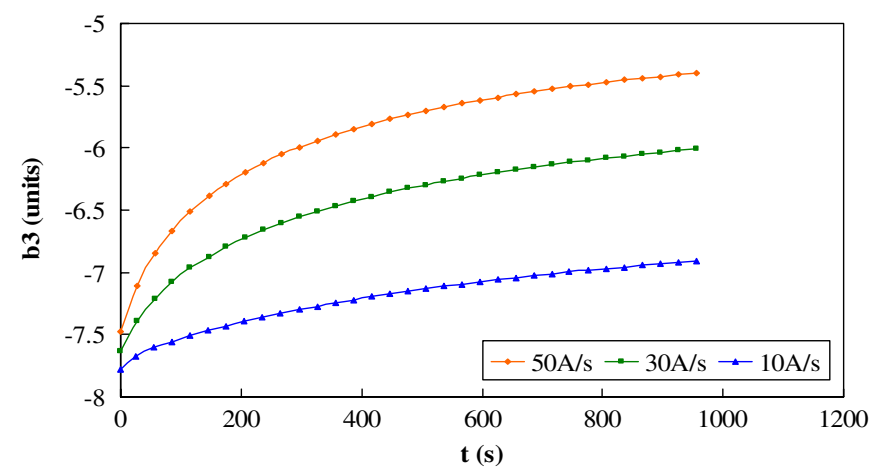

FIG. 3. (Color) MB2598 aperture $2 \mathrm{~b}_{3}$ decay for standard measurement LHC cycles with different precycle ramp rates. 
observed that the decay amplitude is a function of precycle ramp rate, and a fast precycle yields a large decay. In addition, the starting point of the decay appears to be different for the three cycles. We therefore focus on these two observed features so as to properly compensate for them in the machine operation by using FiDeL.

\section{EFFECT OF FINITE MEASUREMENT TIME AT INJECTION}

The different decay starting point is an effect related to the measurement technique and not to a magnet effect. As mentioned earlier, the rotating coils used to take these data perform a measurement in approximately $30 \mathrm{~s}$. In addition, a measurement delay is intentionally introduced at the beginning of the injection plateau to make sure that constant current is reached after the parabolic roll-off from the ramp. This results in a blind time at the beginning of the measurement series, during which a part of the initial decay is lost. The first points of the decay series of Fig. 3 are thus only close to, and not exactly at, the start of the decay.

Further on, observing that the decay amplitude is a function of the precycle ramp rate, it is easy to understand how the blind time at the beginning of the injection plateau leads to an apparent different decay starting point for the three cycles. To demonstrate this feature, we have applied the decay model presented in [3] to each curve and extrapolated backwards in time as shown in Fig. 4. At a time of $26 \mathrm{~s}$ before the first data point, compatible with the measurement time, the standard deviation between the curves is less than 0.02 units, which is of the same order as the reproducibility of the rotating coils. Assuming that this is the real start of the injection, the three curves have in practice the same starting point. In conclusion, we can safely state that the precycle ramp rate has no effect on the value of sextupole at the beginning of injection, as expected.

For the analysis of the influence of precycle ramp rate on decay, this measurement artifact has been removed from the measured curves of the type shown in Fig. 3 using the

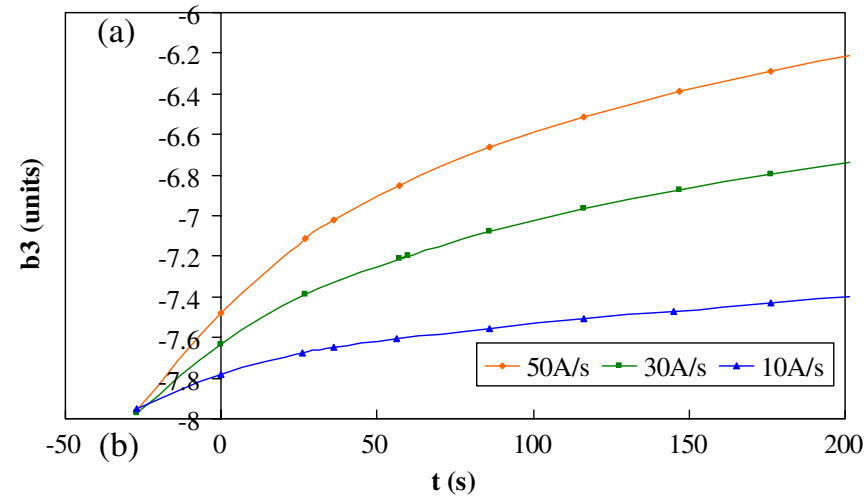

FIG. 4. (Color) Extrapolation of the decay at the beginning of injection. procedure described above and in Fig. 4. The corrected data was then modeled to augment our scaling for dynamic components, as described below. We will show later the influence and correction of the decay during the blind time also on data taken in steady state during loadlines.

\section{THE ANALYTICAL OPERATION CYCLE MODEL}

It has been shown several times that the decay amplitude is related to the decay of the so-called boundary induced coupling currents [5]. Because of the electromagnetic properties of the Rutherford cable, the amplitude of this current imbalance during the operation is assumed (for simplicity) to be proportional to the charging and discharging of an R-L circuit at a certain time during the operation cycles. During the ramp, this circuit is charged and discharged during constant transport current excitation. By computing the actual current amplitude at the end of the injection plateau and taking the recent operation history into account, a value proportional to the sextupole snapback is obtained. The functional relation between operation cycle variations and snapback is given by Eq. (1):

$$
f_{i}=\left\{\begin{array}{l}
\dot{B}_{i}\left(1-e^{-\left(\Delta t_{i} / \tau\right)}\right) e^{\left(\sum_{n=1}^{m} \Delta t_{n}\right) / \tau} \ldots \dot{I} \neq 0 \\
0 \ldots \dot{I}=0 .
\end{array}\right.
$$

It must be stated that the formalism applied is in close connection with the current diffusion model of the twowire Rutherford-type cable simulation of [6,7]. In contrast to this model, where the current imbalance in the two-wire model is given by a sum over current component, each of them linked to a time constant, we approximate the problem with only one amplitude and time constant. Thus, we can take a first order approximation of Krempasky's current diffusion model. The function $f_{i}$ is a representative of the current amplitude in a Rutherford-type cable, which is established during a charging period in the operation cycle sequence of the duration $\Delta t_{i}$. The amplitude then decays during the following operation cycle sequences. This decay lasts over the total sum of time intervals $\Delta t_{n}$.

\section{SCALING OF DECAY AND SNAPBACK WITH PRECYCLE RAMP RATE}

The decay amplitude is known to be affected by the precycle flattop current $\left(I_{\mathrm{FT}}\right)$, the precycle flattop time $\left(t_{\mathrm{FT}}\right)$, and preparation time ( $\left.t_{\text {preparation }}\right)$ according to the definitions shown graphically in Fig. 5, and as discussed in [3]. Here we introduce an additional parameter to the scaling, the precycle ramp rate $(d I / d t)$, also indicated in Fig. 5. Note that the current $I_{\text {preparation }}$ is taken to be the standard minimum excitation current of the magnets and is the current at which the magnets are excited when on standby.

Figures 6 and 7 show a summary of the dependence of the decay amplitude, defined as the change in harmonic 


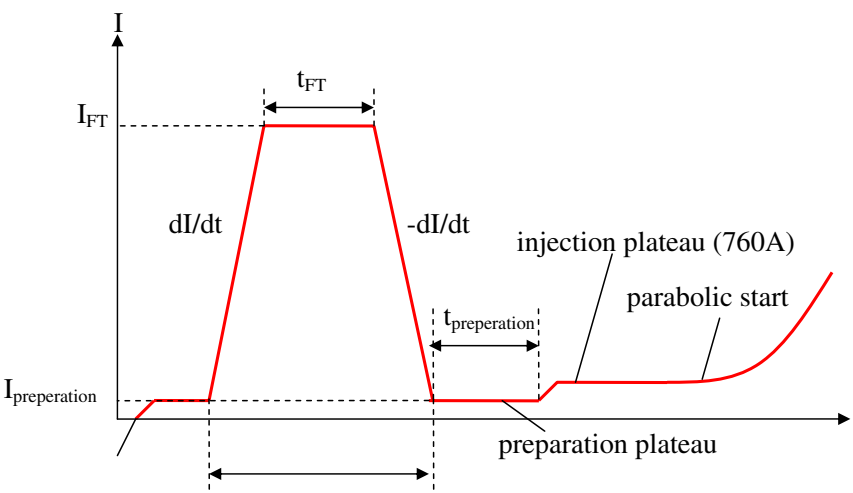

FIG. 5. (Color) Definition of the parameters affecting decay amplitude during LHC injection.

between the beginning and end of injection, on the precycle $I_{\mathrm{FT}}$ and ramp rate $d I / d t$. Figures are reported for the first two allowed harmonics (sextupole $b_{3}$ and decapole $b_{5}$ ), for all LHC dipoles measured, normalized by the value measured in reference conditions (i.e. for a reference precycle at $50 \mathrm{~A} / \mathrm{s}$ ). The data for the dependence on the precycle flattop current is the same as presented in [3], after the correction for the blind time described earlier is applied. The measured magnets were chosen at random spread and, where possible, the choice was spread over the three manufacturers.
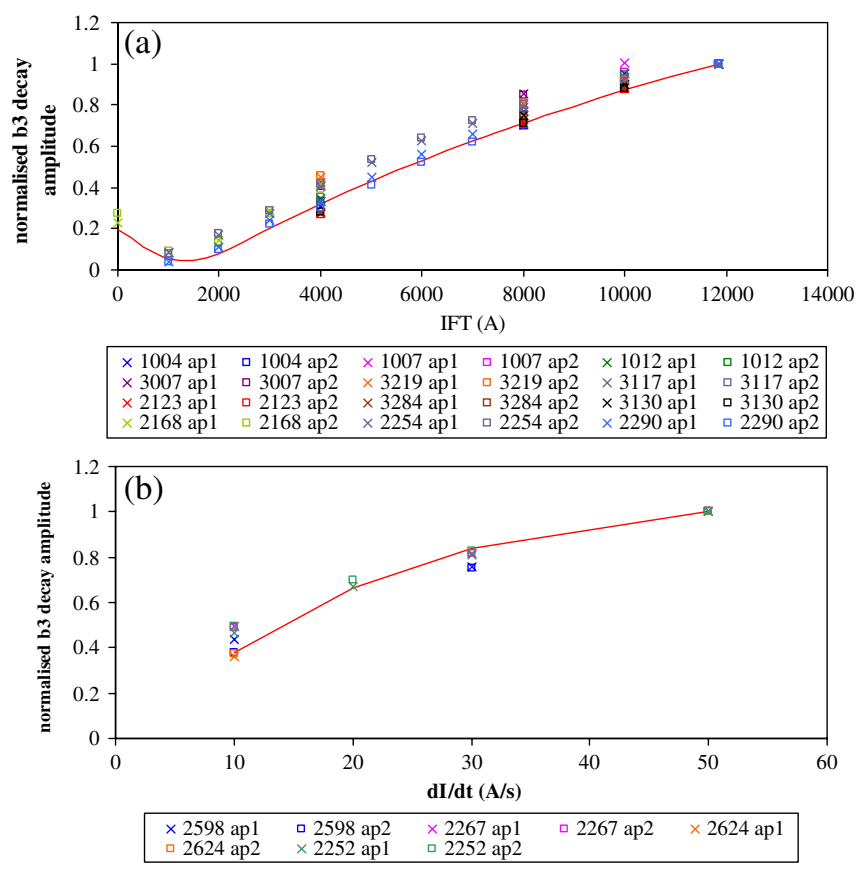

FIG. 6. (Color) (a) The normalized $b_{3}$ decay amplitude and the FiDeL model in red vs the precycle flattop current. (b) The normalized $b_{3}$ decay amplitude and the FiDeL model in red vs the precycle ramp rate. Note: The labeling in the key denotes the magnet number and aperture. The decay amplitude is normalized to $I_{\max }=11850 \mathrm{~A}$. $I_{\mathrm{FT}}=0$ denotes no precycle, i.e., a cycle directly after a quench.

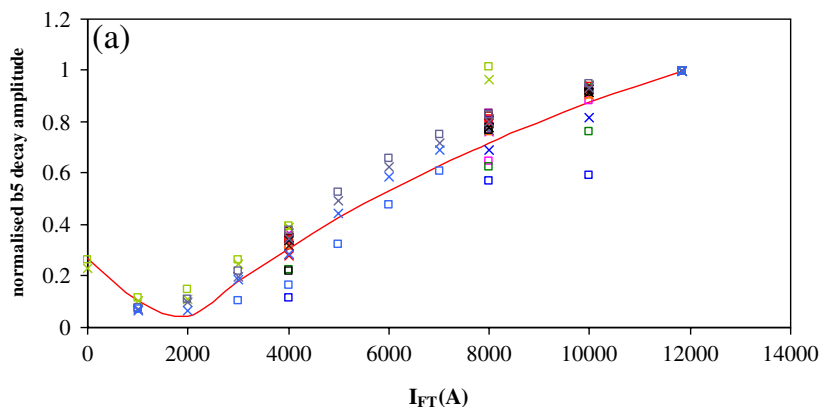

$\times 1004$ ap1 $\square 1004$ ap2 $\times 1007$ ap1 $\square 1007$ ap2 $\times 1012$ ap1 $\square 1012$ ap2 $\times 3007$ ap1 $\square 3007$ ap2 $\times 3219$ ap1 $\square 3219$ ap2 $\times 3117$ ap1 $\square 3117$ ap2 $\times 2123$ ap1 $\square 2123$ ap2 $\times 3284$ ap1 $\square 3284$ ap2 $\times 3130$ ap1 $\square 3130$ ap 2

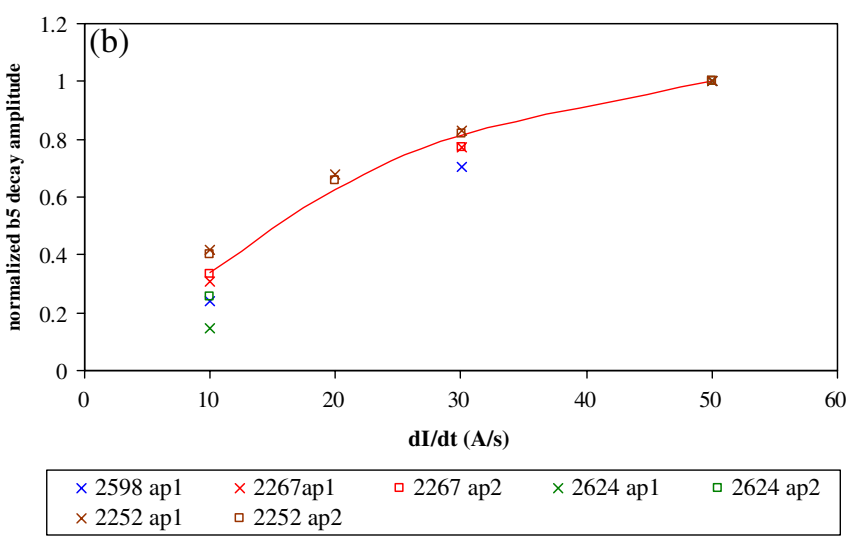

FIG. 7. (Color) (a) The normalized $b_{5}$ decay amplitude and the FiDeL model in red vs the precycle flattop current. (b) The normalized $b_{5}$ decay amplitude and the FiDeL model in red vs the precycle ramp rate. Note: The labeling in the key denotes the magnet number and aperture. The decay amplitude is normalized to $I_{\max }=11850 \mathrm{~A}$. $I_{\mathrm{FT}}=0$ denotes no precycle, i.e., a cycle directly after a quench.

The precycle ramp rate has an appreciable effect on the decay amplitude, comparable to that of the flattop current.

After normalizing each term with the effect of the precycle ramp rate can be included in FiDeL by using Eq. (1) and by modifying the first term of Eq. (16) in [3] as follows:

$$
\begin{aligned}
& \delta_{n}=\delta_{\mathrm{std}}\left|\frac{\frac{d I}{d t}}{\frac{d I}{d t} \operatorname{ref}} \frac{E_{0}^{n}-E_{1}^{n} e^{-I_{\mathrm{FT}} /\left[\tau_{E}^{n} \cdot(d I / d t)\right]}}{E_{0}^{n}-E_{1}^{n} e^{-I_{\mathrm{FT}}^{\mathrm{std}} /\left[\tau_{E}^{n} \cdot(d I / d t) \mathrm{ref}\right]}}\right| \\
& \times \frac{T_{0}^{n}-T_{1}^{n} e^{-t_{\mathrm{FT}} / \tau_{T}^{n}}}{T_{0}^{n}-T_{1}^{n} e^{-t_{\mathrm{FT}}^{\mathrm{st}} / \tau_{T}^{n}}} \frac{P_{0}^{n}-P_{1}^{n} e^{-t_{\text {preparation }} / \tau_{P}^{n}}}{P_{0}^{n}-P_{1}^{n} e^{-t_{\text {preparation }}^{\mathrm{std}} / \tau_{P}^{n}}} .
\end{aligned}
$$

The first term describes the dependency of the decay amplitude on the precycle flattop current and on the precycle ramp rate. This equation resulted from modeling the decay amplitude effect as an R-L circuit [8-10] excited by the subsequent ramp-ups and ramp-downs. In this equation, $I_{\mathrm{FT}}$ is the current reached at the precycle flattop, $t_{\mathrm{FT}}$ is the flattop duration, $t_{\text {preparation }}$ is the preparation time, and $\frac{d I}{d t}$ is the precycle ramp rate. $I_{\mathrm{FT}}^{\mathrm{std}}, t_{\mathrm{FT}}^{\mathrm{std}}$, and $t_{\text {preparation }}^{\mathrm{std}}$ are the normalization parameters which are the values of the pa- 
TABLE I. The fitting parameters of Eq. (2).

\begin{tabular}{lcccc}
\hline \hline & Dimension & $\mathrm{b}_{1}$ & $\mathrm{~b}_{3}$ & $\mathrm{~b}_{5}$ \\
\hline$E_{0}$ & $\ldots$ & 0.19343 & 0.60977 & 0.23005 \\
$E_{1}$ & $\ldots$ & 0.17531 & 0.67227 & 0.265891 \\
$\tau_{E}$ & $\mathrm{~s}$ & 150 & 286 & 233 \\
$T_{0}$ & $\ldots$ & & -5.28838 & \\
$T_{1}$ & $\ldots$ & & -1.37640 & \\
$\tau_{T}$ & $\mathrm{~s}$ & & 509 & \\
$P_{0}$ & $\ldots$ & & -1.16444 & \\
$P_{1}$ & $\ldots$ & & 0.50944 & \\
$\tau_{P}$ & $\mathrm{~s}$ & & 307 & \\
$\frac{d I}{d t}$ ref & $\mathrm{A} / \mathrm{s}$ & 50 & 50 & 50 \\
$I_{\mathrm{FT}}^{\text {std }}$ & $\mathrm{A}$ & 11850 & 11850 & 11850 \\
$t_{\mathrm{FT}}^{\text {std }}$ & $\mathrm{s}$ & 1000 & 1000 & 1000 \\
$t_{\text {preparation }}^{\text {std }}$ & $\mathrm{s}$ & 0 & 0 & 0 \\
\hline \hline
\end{tabular}

rameters in the standard series LHC cycle measurements, already defined in [3]. $\frac{d I}{d t}$ ref is the precycle current ramp rate in the series measurements $(50 \mathrm{~A} / \mathrm{s})$, assumed to be identical for ramp-up and ramp-down. $E_{0}, E_{1}, \tau_{E}, T_{0}, T_{1}$, $\tau_{T}, P_{0}, P_{1}$, and $\tau_{P}$ are the fitting parameters.

It should be noted that this equation incorporates several effects that are dependent on several parameters and in itself is a simplification of an equation that is based on a series [8-10]. So as to keep the model simple but effective, it was decided to limit the number of terms in the equation. Whilst this works well in practice, the drawback is that the determination of the fitting parameters is dependent on the way the equation is allowed to converge. It should also be pointed out that the parameters of Table I are only valid for the ranges indicated in Figs. 6 and 7.

Using the model equation (2) on the data of Figs. 6 and 7, we obtain the best fit parameters reported in Table I. Based on the model results, we expect that the average decay amplitude in the LHC for a precycle at $10 \mathrm{~A} / \mathrm{s}$ will be of about 0.9 units of sextupole $b_{3}$, vs an average of about two units established during series measurements with a precycle at $50 \mathrm{~A} / \mathrm{s}$. This is a significant effect. Measurements (not reported) also show that the decay of the main field becomes negligible for a precycle ramp rate of $10 \mathrm{~A} / \mathrm{s}$. The fitting parameters of Eq. (2) are shown in Table I.

\section{CORRECTION OF STATIC (LOADLINE) DATA}

The data taken during loadline measurements on which the static field components are based were reexamined after the finding discussed above. The loadline measurement is taken at a number of constant current plateaus (about 20 along a ramp), a few seconds after reaching each plateau. For each plateau, two data points are taken and averaged to produce curves of static harmonics vs current. Although the static field component should not depend on the precycle ramp rate, we expected that the decay on each plateau could affect the final average (over a

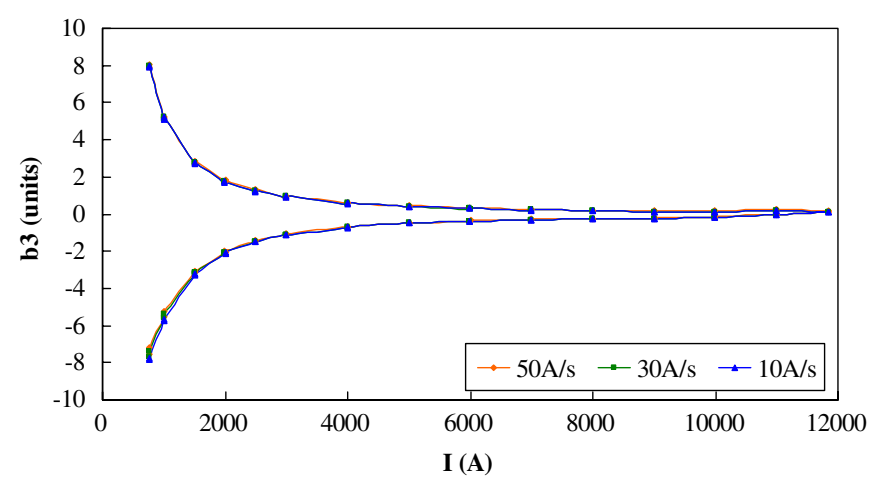

FIG. 8. (Color) Loadline measurement (MB2598 aperture 2) with different precycle ramp rates.

finite measurement time) and induce an artificial dependence. We have verified this hypothesis, performing three loadline measurements on one dipole, with a different precycle ramp rate. The results are shown in Fig. 8, reporting $b_{3}$ vs current.

To examine in detail the differences observed, we report in Fig. 9 a close-up of the single measurements on the plateaux around $760 \mathrm{~A}$. We observe that between the two measurements on each plateau there is indeed a decay that depends on the precycle ramp rate and reduces with increasing current. Using the technique described earlier, it is possible to extrapolate the decay back to where the plateau is assumed to start, thus correcting for the different decay amplitudes at different currents. Once corrected, the load-
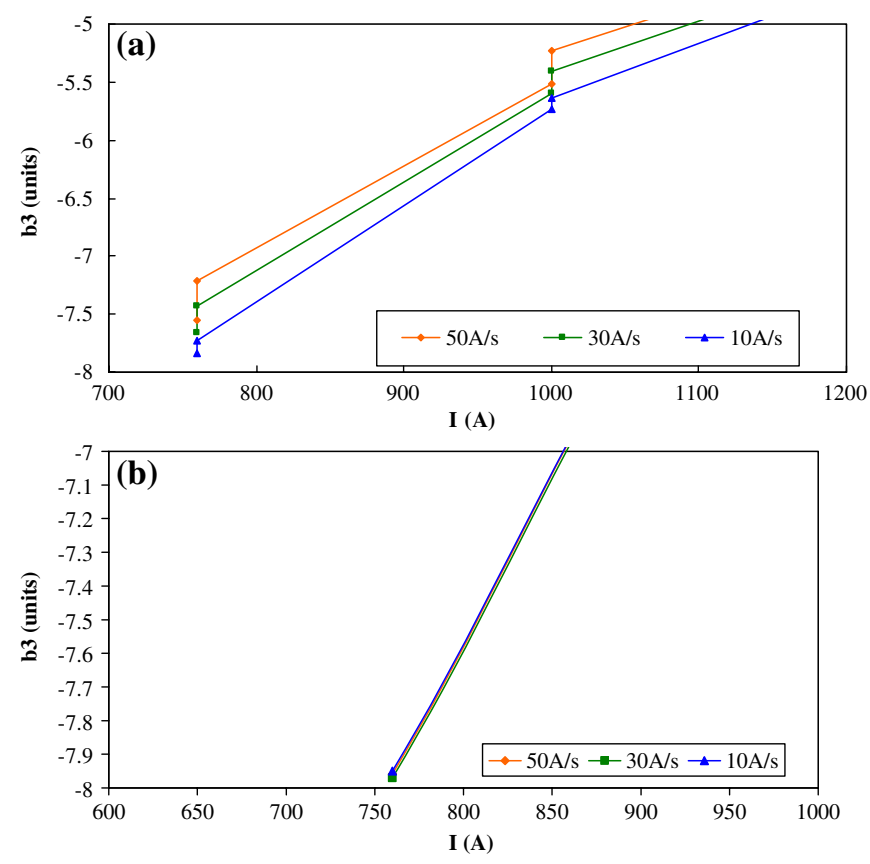

FIG. 9. (Color) (a) Same as Fig. 8. Detail of single measurements for the loadline data reported in Fig. 8 (MB2598 aperture 2) with different precycle ramp rates around $760 \mathrm{~A}$. (b) Loadline measurements after correction. 


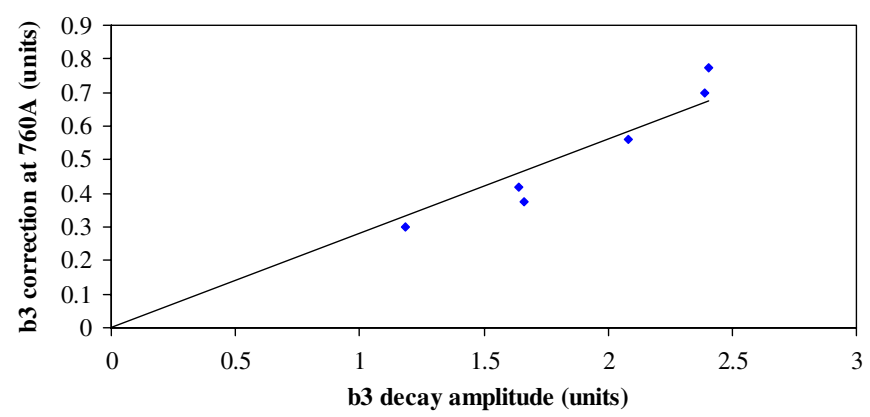

FIG. 10. (Color) The $b_{3}$ correction at $760 \mathrm{~A}$ vs the $\mathrm{b}_{3}$ decay amplitude at $760 \mathrm{~A}$.

line measurements become practically identical, see the bottom of Fig. 9. The maximum standard deviation between the three corrected cycles is 0.03 units, negligibly small. Without the above correction, the $b_{3}$ value at injection would have been taken -7.37 units, i.e., the average of the two points at $760 \mathrm{~A}$ for the precycle at $50 \mathrm{~A} / \mathrm{s}$. The correct extrapolation yields $a b_{3}$ value of -7.96 units, i.e., a difference $\delta \mathrm{b}_{3}$ of 0.59 units which is significant. This simple study shows that a correction is hence required also for all static data from series measurements.

To avoid reprocessing all series measurements, which would be a very tedious and time consuming process, we decided to apply a global correction to the average of cold data. Detailed measurements of a few magnets, fully characterized as described above, provide a correction $\delta(I)$ to be applied to loadline (static) data as a function of current. To make $\delta(I)$ representative of the average of the population of cold tested magnets, we note that there must be a correlation between the correction to be applied and the amplitude of decay in each magnet. This correlation is demonstrated in Fig. 10 for $b_{3}$. The decay amplitude, in turn, is readily available for all magnets cold tested. The average $b_{3}$ decay for all magnets cold tested is 2.05 units at $760 \mathrm{~A}$, which corresponds to a correction $\delta(760)$ of 0.56 units. Using the correlation of Fig. 10, it is hence possible to scale the correction $\delta(I)$ at each current to produce values representative of the average of the whole magnet

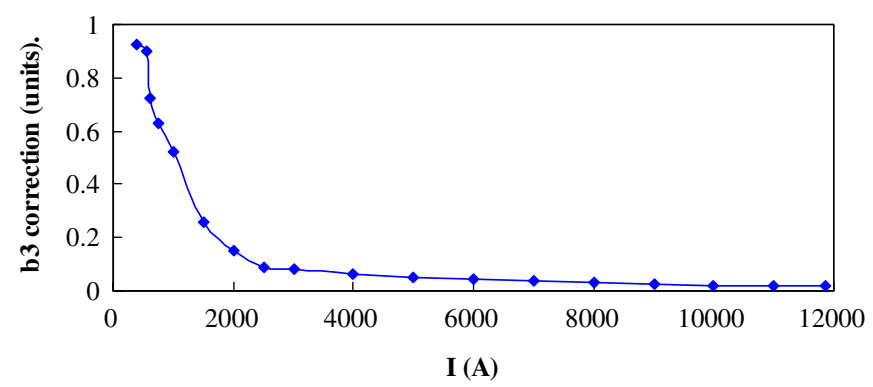

FIG. 11. (Color) The $b_{3}$ correction to be implemented on the loadline ramp-up measurements of the LHC dipole magnet population. The values from the plot above are subtracted from the loadline data. population. The final result is shown in Fig. 11 for the sextupole $b_{3}$.

\section{CONCLUSION}

We have examined the effect of the precycle ramp rate on the measured field quality of the LHC superconducting dipole magnets, both in the static and dynamic domain. In the range of measurement and operation, the precycle ramp rate has no impact on the static field, but a very significant effect on the decay amplitude at injection. This happens, in our present understanding, because the time constant of the decay as modeled in [3] is comparable to the time for the magnet to ramp down to minimum current and back up to injection current. We have included this effect in the FiDeL model of decay, to be able to extrapolate properly from series measurements to the LHC operating conditions. In practice, we expect that the decay amplitude (and snapback) during normal machine operation is about half of what was found in series measurements.

Based on these findings, and improved understanding, we have revisited the assumption of constant magnetic field that was used to analyze the measurements taken on the current plateaux of a loadline cycle, which form the basis of the static part of the FiDeL model. Neglecting the inevitable field changes that take place even during measurements at constant current gives a significant error on the evaluated static field, e.g., in the LHC dipoles of the order of 0.6 units of $b_{3}$ at injection. The decay model was applied to obtain a correction of the loadline measurements to be applied to the average of the magnet population.

[1] O. Brüning, P. Collier, P. Lebrun, S. Myers, R. Ostojic, J. Poole, and P. Proudlock, CERN Report No. CERN-2004003, 2004.

[2] N. Sammut, L. Bottura, and J. Micallef, Phys. Rev. ST Accel. Beams 9, 012402 (2006).

[3] N. Sammut, L. Bottura, P. Bauer, T. Pieloni, and J. Micallef, Phys. Rev. ST Accel. Beams 10, 082802 (2007).

[4] J. Billan, L. Bottura, M. Buzio, G. D’Angelo, G. Deferene, O. Dunkel, P. Legrand, A. Rijllart, A. Siemko, P. Sievers, S. Schloss, and L. Walckiers, IEEE Trans. Appl. Supercond. 10, 1422 (2000).

[5] A. Verweij, Proceedings of the 12th International Cryogenic Materials Conference, Portland, Oregon, USA, 1997 (Plenum, New York, 1997), pp. 1059-1068.

[6] L. Krempasky and C. Schmidt, Appl. Phys. Lett. 66, 1545 (1995).

[7] L. Krempasky and C. Schmidt, Cryogenics 39, 23 (1999).

[8] Thomas Schreiner, Ph.D. thesis, Vienna University of Technology, 2002.

[9] M. Haverkamp, Ph.D. thesis, Twente University, Enschede, The Netherlands, 2003.

[10] M. Schneider, Ph.D. thesis, Institute for Experimental Physics, Vienna, Austria, 1998. 